\title{
Charge Catastrophe and Dielectric Breakdown During Exposure of Organic Thin Films to Low-Energy Electron Radiation
}

\author{
A. Thete, ${ }^{1,2}$ D. Geelen, ${ }^{1}$ S. J. van der Molen, ${ }^{1}$ and R. M. Tromp ${ }^{1,3}$ \\ ${ }^{1}$ Leiden University, Huygens-Kamerlingh Onnes Laboratory, P.O. Box 9504, 2300 RA Leiden, The Netherlands \\ ${ }^{2}$ Advanced Research Center for Nanolithography, Science Park 102, 1098 XG Amsterdam, The Netherlands \\ ${ }^{3}$ IBM T.J. Watson Research Center, 1101 Kitchawan Road, Yorktown Heights, New York 10598, USA
}

(Received 20 February 2017; published 28 December 2017)

\begin{abstract}
The effects of exposure to ionizing radiation are central in many areas of science and technology, including medicine and biology. Absorption of UV and soft-x-ray photons releases photoelectrons, followed by a cascade of lower energy secondary electrons with energies down to $0 \mathrm{eV}$. While these low energy electrons give rise to most chemical and physical changes, their interactions with soft materials are not well studied or understood. Here, we use a low energy electron microscope to expose thin organic resist films to electrons in the range $0-50 \mathrm{eV}$, and to analyze the energy distribution of electrons returned to the vacuum. We observe surface charging that depends strongly and nonlinearly on electron energy and electron beam current, abruptly switching sign during exposure. Charging can even be sufficiently severe to induce dielectric breakdown across the film. We provide a simple but comprehensive theoretical description of these phenomena, identifying the presence of a cusp catastrophe to explain the sudden switching phenomena seen in the experiments. Surprisingly, the films undergo changes at all incident electron energies, starting at $\sim 0 \mathrm{eV}$.
\end{abstract}

DOI: 10.1103/PhysRevLett.119.266803

The interaction of ionizing radiation with matter is of vast scientific and technological (including biological and medical) importance. The interaction of UV and $\mathrm{x}$-ray photons with matter is mediated by photoelectrons, as well as secondary electrons with a broad energy distribution that induce chemical changes in the material, be it a polymer, organic or inorganic hybrid, biological tissue, or even DNA. But these complex processes are hard to disentangle, as photon illumination sets the entire electron cascade in motion at once, without the possibility of discerning the role of electrons with different energies. As a result, the interaction of low energy electrons (LEEs) with soft matter is not well understood. Here, we focus primarily on the interaction of low energy electrons with polymethylmethacrylate (PMMA) and related resist materials as used in extreme ultraviolet (EUV) lithography [1] to obtain a new understanding of key processes at low electron energies.

In a low energy electron microscope [2] (LEEM) a sample is illuminated with electrons with adjustable 0-100 eV energy [3]. We use LEEM to expose thin PMMA films, monitoring changes both after and during exposure [4]. The radiation chemistry of PMMA and related materials has been well studied, and there is consensus that irradiation causes scission of the main chains and removal of side groups [5-10]. Here, we identify key physical processes largely ignored in the literature: resist charging, exposure-induced changes in conductivity and secondary electron emission, and dielectric breakdown. We present a simple quantitative theory describing our data, identifying a cusp catastrophe [11] causing the instabilities seen during exposure. Even electrons with near-zero energy change the resist, suggestive of dissociative electron attachment processes [12] commonly neglected in resist modeling. Our results provide new insights into LEE interactions in a broader sense, deepening our knowledge of the interaction of ionizing radiation with soft matter.

Experiments were performed in the ESCHER LEEM facility [4] at Leiden University. The sample is immersed in an electrostatic field of $\sim 100 \mathrm{kV} / \mathrm{cm}$, slowing the $15 \mathrm{keV}$ electrons produced by the gun to tunable $0-100 \mathrm{eV}$ incident energy, $E_{0}$. Secondary electrons leaving the sample are extracted by this field, and can never return [2-4]. The experiment is schematically shown in Fig. 1(b).

Figure 1(a) shows a $20 \mathrm{~nm}$ PMMA film exposed to varying electron energies, currents, and doses [4]. Each bright spot represents a single exposure with $\sim 5 \mu \mathrm{m} \varnothing$. Between exposures the beam is blanked, and the sample position is advanced. With all exposures complete the sample is developed in 1:3 isopropyl alcohol:methyl isobutyl-ketone for $1 \mathrm{~min}$, and viewed under an optical microscope. We find an apparent energy threshold below which the resist is not exposed. This threshold depends on beam current, increasing from $\sim 15 \mathrm{eV}$ at $0.05 \mathrm{nA}$, to $\sim 18 \mathrm{eV}$ at $2 \mathrm{nA}$, but not on dose. We will show that this threshold shift is not related directly to electron energy, but to charging of the resist, which depends on electron energy and current, electrical conductivity of the resist, and secondary electron emission (SEE). Below threshold the PMMA surface accumulates sufficient negative charge to reflect the incident electrons and prevent them from reaching the sample. Figure 1(c) shows some of the elementary 


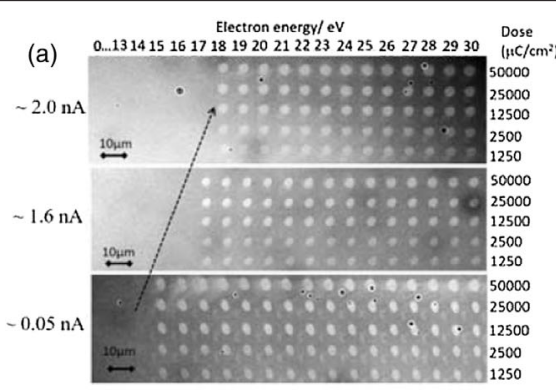

(b)

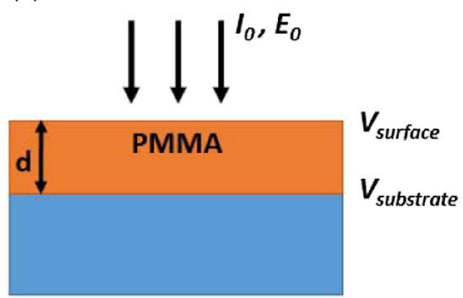

(c)

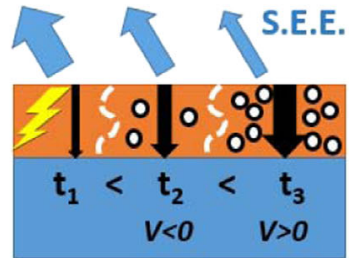

FIG. 1. (a) PMMA exposures as a function of electron current, energy, and dose. At each current $(0.05,1.6$, and $2.0 \mathrm{nA})$ we find an exposure threshold which does not depend on dose. PMMA thickness $20 \pm 4 \mathrm{~nm}$, spin-coated onto a Si substrate. (b) An electron beam

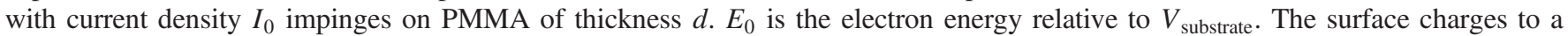
potential $V_{\text {surface. }}$. The charging voltage $V$ is defined as $V=V_{\text {substrate }}-V_{\text {surface }}$ (c) Schematic of elementary processes, including electrical breakdown (time $t_{1}$ ), increasing trap creation (white dots at $t_{2}$ and $t_{3}$ ), decreasing SEE and increasing conductance during exposure, $V$ switching sign between $t_{2}$ and $t_{3}$. These processes depend on experimental parameters that change over time.

processes, i.e., surface charging, dynamic changes in PMMA conductance and secondary electron emission, and the balance between them. At short times charging can be so severe as to give rise to dielectric breakdown across the PMMA film.

Figures 2(a)-2(e) present energy spectra of electrons reflected and/or emitted by the sample during exposure [13], for $E_{0}$ from $14-30 \mathrm{eV}(0.25 \mathrm{nA}, 5 \mu \mathrm{m} \varnothing)$. Electron intensity is shown vs energy and time. $E_{0} \leq 14 \mathrm{eV}$ [Fig. 2(a)] yields only specularly reflected electrons, implying that the surface charges to the beam energy, and all electrons are backreflected before reaching the sample. At $E_{0}=15 \mathrm{eV}$ Fig. 2(b) first shows a narrow spectrum, as the electrons are decelerated to near-zero energy by accumulation of negative surface charge. The spectrum width increases over time as negative charge diminishes, thereby increasing the landing energy, $E_{\text {land }}$. In Fig. 2(c), the initial signal at $\sim 31 \mathrm{eV}$ exceeds $E_{0}=20 \mathrm{eV}$, i.e., the incident electrons are accelerated from 20 to $31 \mathrm{eV}$ due to accumulation of positive charge. $E_{\text {land }}$ slowly decreases, followed by a sudden drop to $\sim 15 \mathrm{eV}$. In Figs. 2(d)-2(e) we again find an initial acceleration of the incident electrons, with a drop of $E_{\text {land }}$ during the first few seconds to $E_{\text {land }} \approx E_{0}+5 \mathrm{eV}$. Then $E_{\text {land }}$ slowly decreases, followed again by a sudden drop near the center of the data sets. Such erratic and unstable behavior cannot be understood in a static picture of electron-PMMA interaction.

To understand the threshold, we define the substrate as one electrode, and the PMMA surface as a second "virtual" electrode on which charge can accumulate, and then flow to the substrate. $V$ is defined as $V_{\text {substrate }}-V_{\text {surface }}$ [Fig. 1(b)]. The current density from surface to substrate is given by the Mott-Gurney law for space-charge-limited conductance [14]:

$$
I(V)= \pm g V^{2}
$$

where $g=9 \varepsilon \mu / 8 d^{3}$ (dielectric constant $\varepsilon$, mobility $\mu$, thickness $d$ ). The minus sign applies for $V<0$.

The Gaussian energy distribution of the electron beam is given by

$$
I_{0}(E)=I_{0} \frac{1}{\sqrt{2 \pi \sigma^{2}}} e^{-\frac{\left(E-E_{0}\right)^{2}}{2 \sigma^{2}}} .
$$

$E_{0}$ is the incident electron energy relative to $V_{\text {substrate }}$, with standard deviation $\sigma=0.11 \mathrm{eV}$ in our experiments. If $V=0$ (no charging), the incident current equals $\int_{0}^{\infty} I_{0}(E) d E$, which for $E_{0}>0.3 \mathrm{eV}$ equals $I_{0}$. However, for typical current densities $I_{0}$ the surface charges to an electronretarding potential $V$, and only electrons with $E>V$
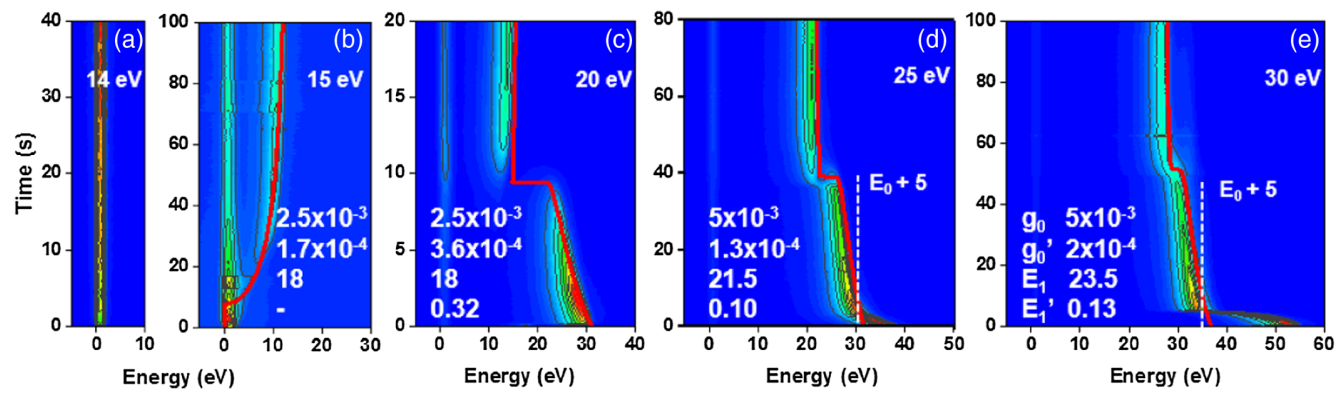

FIG. 2. (a)-(e) Electron energy spectra during exposure for $E_{0}=14,15,20,25$, and $30 \mathrm{eV}$. The energy scale is a loss scale, with elastic electrons at zero. Thus, the highest energy at which signal is observed (i.e. the cut-off of the secondary electrons) is a direct measure of

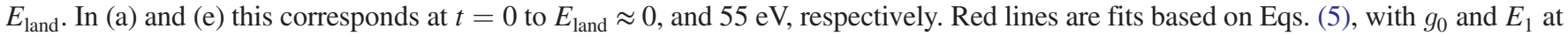
$t=0$, and time derivatives $g_{0}^{\prime}$ and $E_{1}^{\prime}$ given in the figures. 
reach the surface. (We take the electron charge $e=1$ for convenience.) Then the net incident current density is $\int_{V}^{\infty} I_{0}(E) d E$. Slower electrons $(E<V)$ never reach the sample. In equilibrium the current through the film equals the net incident current:

$$
\frac{I(V)}{I_{0}}= \pm g_{0} V^{2}=\int_{V}^{\infty} \frac{1}{\sqrt{2 \pi \sigma^{2}}} e^{-\frac{\left(E-E_{0}\right)^{2}}{2 \sigma^{2}}} d E,
$$

where $g_{0}=g / I_{0}$. The PMMA surface charges to a potential $V_{\text {eq }}$ that satisfies Eq. (3). Figure 3(a) plots the left-hand side of Eq. (3) vs $V$ for $g_{0}=0.0045$ (blackdashed curve), and the right-hand side (blue lines) for $E_{0}=10,15$, and $20 \mathrm{eV}(\sigma=0.11 \mathrm{eV})$. Equation (3) is satisfied where the black and blue lines intersect (arrows). For $E_{0}=10 \mathrm{eV}$ we find $V_{\text {eq }} \approx 10 \mathrm{~V}$ (red arrow). About half the electrons reach the sample with near-zero energy, while the other half is reflected back into the vacuum. For $E_{0}=15 \mathrm{eV}, V_{\text {eq }} \approx 14.5 \mathrm{~V}$ (black arrow) and $\sim 95 \%$ of the electrons reach the sample with $E_{\text {land }} \approx 0.25 \mathrm{eV}$. For

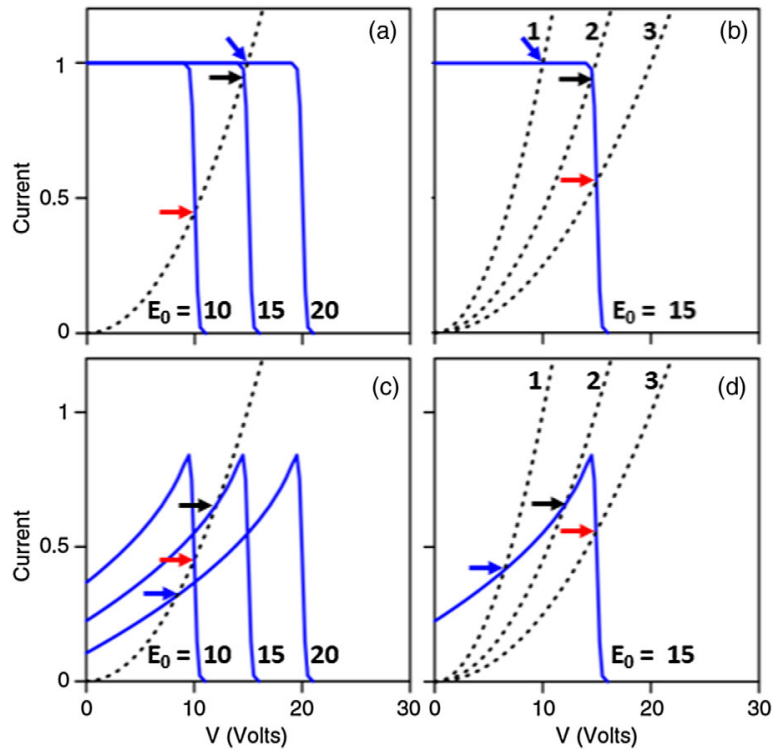

FIG. 3. (a) Blue lines: Normalized electron current vs $V$ without secondary electrons for $E_{0}=10,15$, and $20 \mathrm{eV}$. Black dash: lefthand side of Eq. (3) with $g_{0}=0.0045$. Equation (3) is satisfied at the intersections of the blue and black lines (arrows). (b) Blue line: as in (a) with $E_{0}=15 \mathrm{eV}$. Black lines: as in (a) with $g_{0}=$ 0.01 (1), 0.0045 (2), and 0.0025 (3). Charging increases with decreasing $g_{0}$, i.e., increasing $I_{0}$. (c) Blue lines: Normalized electron current vs $V$ with secondary electrons [right-hand side of Eq. 5(b)] for $E_{0}=10,15$, and $20 \mathrm{eV}, \alpha=0.5$, and $E_{1}=25 \mathrm{eV}$. Black dashed curve: left-hand side of Eq. (3) with $g_{0}=0.0045$. For $E_{0}=10 \mathrm{eV}, V_{\mathrm{eq}}$ has not changed relative to (a), but for $E_{0}=$ 15 and $20 \mathrm{eV}$ it has decreased markedly (arrows). (d) Blue line: as in (c) with $E_{0}=15 \mathrm{eV}$. Black lines: as in (c) with $g_{0}=0.01$ (1), 0.0045 (2), and 0.0025 (3). For the highest beam current (3) $V_{\text {eq }}$ has changed little compared to (b). For (1) and (2) $V_{\text {eq }}$ has decreased markedly.
$E_{0}=20 \mathrm{eV}, V_{\text {eq }} \approx 14.9 \mathrm{eV}$ (blue arrow) and all electrons reach the sample with $E_{\text {land }} \approx 5.1 \mathrm{eV}$. Figure $3(\mathrm{~b})$ plots the right-hand side of Eq. (3) for $E_{0}=15 \mathrm{eV}$ (blue), and the black-dashed lines are for $g_{0}=0.01,0.0045$, and 0.0025 . Upon decreasing $g_{0}$ (increasing $I_{0}$ ), $V_{\text {eq }}$ shifts to the right. For the highest $I_{0}$ (lowest $g_{0}$ ) the surface charges to $E_{0}$ (red arrow), for medium $I_{0}$ to just below $E_{0}$ (black arrow), and for the lowest $I_{0}$ to $\sim 5 \mathrm{~V}$ below $E_{0}$ (blue arrow). The threshold shifts upwards with $I_{0}$, in accordance with Fig. 1(a).

Equation (3) does not account for secondary electrons leaving the sample. The SEE coefficient as a function of incident electron energy, $\delta_{s}(E)$, has been studied extensively [15-23], but is not well characterized below $100 \mathrm{eV}$. We approximate $\delta_{s}(E)$ by

$$
\delta_{s}(E)=\left(\frac{E}{E_{1}}\right)^{\alpha} .
$$

$E_{1}$ is the energy for which $\delta_{s}(E)=1, \alpha$ falls in the range $0.5-1.5$ (the value of $\alpha$ is not critical; see the Supplemental Material [24]).

Secondaries leaving the sample reduce the net electron current reaching the sample; the weight of each incident electron is reduced by $\delta_{s}(E)$. For incident energy $E$ and charging potential $V, E_{\text {land }}=E-V$, and the reduced electron weight is $\left[1-\delta_{s}\left(E_{\text {land }}\right)\right]$, changing Eq. (3) to

$\frac{I(V)}{I_{0}}= \pm g_{0} V^{2}=\int_{V}^{\infty} \frac{1}{\sqrt{2 \pi \sigma^{2}}}\left(1-\left(\frac{E-V}{E_{1}}\right)^{\alpha}\right) e^{-\frac{\left(E-E_{0}\right)^{2}}{2 \sigma^{2}}} d E$.

Figure 3(c) shows the left-hand side of Eq. (5a) (blackdashed curve, $g_{0}=0.0045$ ), and the right-hand side for $E_{0}=10,15$, and $20 \mathrm{eV}$, taking $E_{1}=25 \mathrm{eV}, \alpha=0.5$. Comparison with Fig. 3(a) shows the importance of including SEE: $E_{0}=10 \mathrm{eV}$ is still well below threshold (red arrow), but $E_{0}=15 \mathrm{eV}$ is well above (black arrow). For $E_{0}=20 \mathrm{eV}, V_{\text {eq }}$ has shifted from $\sim 15 \mathrm{eV}$ in Fig. 3(a) to $\sim 8 \mathrm{eV}$ in Fig. 3(c) (blue arrow). In Fig. 3(d) we again find this downward shift in $V_{\text {eq }}$ (arrows) compared to Fig. 3(b).

If the electron energy distribution is infinitely narrow ( $\delta$ function), Eq. (5a) simplifies to

$$
\frac{I(V)}{I_{0}}= \pm g_{0} V^{2}=1-\left(\frac{E_{0}-V}{E_{1}}\right)^{\alpha} .
$$

With $\sigma=0.11 \mathrm{eV}$ this excellent approximation is used in the following. Figure 4(a) shows the left-hand side of Eq. (5b) (black-dashed curve, $g_{0}=0.0025$ ), for $-30<V<30$. The blue lines show the right-hand side of Eq. (5b) for $E_{0}=25 \mathrm{eV}$, and $E_{1}=20,25$, and $30 \mathrm{eV}$, $\alpha=0.5$. For $E_{1}=30 \mathrm{eV}$ there is one solution (blue arrow) with $E_{0}-V_{\text {eq }}=E_{\text {land }}<E_{0}$. For $E_{1}=20 \mathrm{eV}$ there is one 

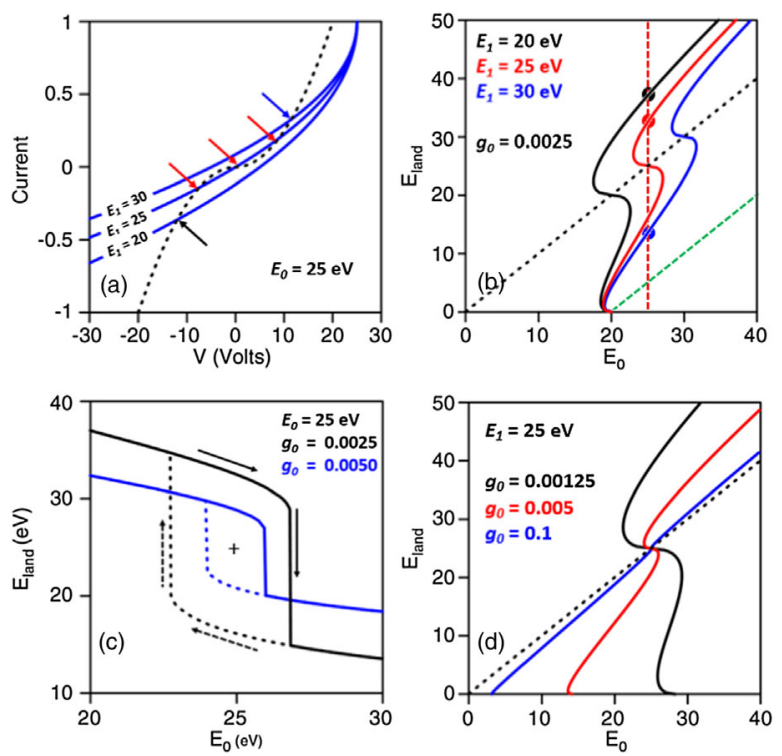

FIG. 4. (a) Black dashed curve: left-hand side of Eq. (5b), $g_{0}=0.0025$. Blue lines: right-hand side of Eq. (5b) for different values of $E_{1}$. Arrows indicate solutions of Eq. (5b). (b) Solutions to Eq. (5b) vs $E_{0}$ for different values of $E_{1} \cdot g_{0}=0.0025$. Green dashed line: no secondary electrons (c) $E_{\text {land }}$ vs $E_{1}$ at $E_{0}=25 \mathrm{eV}$. As $E_{1}$ increases (solid lines), $E_{\text {land }}$ drops abruptly at a critical value. Reducing $E_{1}$, the landing energy jumps back up at a much lower value (dashed lines). This hysteresis, characteristic of a cusp catastrophe, closes as $g_{0}$ increases (blue lines). (d) Solutions to Eq. (5b) vs $E_{0}$ for different values of $g_{0}$. $E_{1}=25 \mathrm{eV}$. This is typical of a cusp catastrophe with parameters $E_{0}$ and $g_{0} . \alpha=0.5$ throughout.

solution (black arrow) of opposite sign $\left(E_{\text {land }}>E_{0}\right)$, corresponding to positive charge accumulation on the surface. Finally, $E_{1}=25 \mathrm{eV}$ has three solutions (red arrows).

Figure 4(b) shows the solutions of Eq. (5b), i.e., $E_{\text {land }}=E_{0}-V_{\text {eq }}$, vs $E_{0}$, with $g_{0}=0.0025$ and $E_{1}=20$ (black), 25 (red), and 30 (blue) eV. Again, at $E_{0}=25 \mathrm{eV}$ (vertical red dash) we find one solution for $E_{1}=20$, and for $E_{1}=30$, and three solutions for $E_{1}=25 \mathrm{eV}$. In each case, $E_{\text {land }}=0$ for $E_{0}<20 \mathrm{eV}$, i.e., the surface charges to the beam energy, independent of $E_{1}$. The curves have an s-shape that depends on $E_{1}$, causing the instabilities in Fig. 2 (see below). When there are no secondary electrons $\left(E_{1} \rightarrow \infty\right)$ the sample also charges to $20 \mathrm{eV}$, beyond which $E_{\text {land }}$ increases linearly with $E_{0}$ (green dash).

Alternatively, we can change $g_{0}$ from 0.00125 [Fig. 4(d), black], to 0.005 (red), and 0.1 (blue). Increasing $g_{0}$ corresponds to increasing conductance, or decreasing $I_{0}$. For $g_{0}=$ 0.1 we find a low threshold of $\sim 3 \mathrm{eV}$, and $E_{\text {land }} \cong E_{0}$. The black dashed line, $E_{\text {land }}=E_{0}$, represents no charging $\left(g_{0} \rightarrow \infty\right)$. For lower $g_{0}$ the threshold increases, in agreement with Fig. 1(a). The $s$ shapes become more pronounced for lower $g_{0}$, i.e., for high resistance and/or high $I_{0}$ there is the possibility of multiple values of $E_{\text {land }}$ over some range of $E_{0}$ centered around $E_{1}$. Generically, Figs. 4(b)-4(d) are emblematic of a so-called cusp catastrophe [11], where one may expect unstable behavior.

Two factors cause the instabilities in Fig. 2: exposureinduced reduction in SEE (increasing $E_{1}$ ), as well as increasing conductance $\left(g_{0}\right)$. First consider SEE, starting with $E_{\text {land }}$ on the upper branch of the black $s$ curve in Fig. 4(d) for $E_{0}=25 \mathrm{eV}$ (black dot), i.e., the only solution available for these initial values. Increasing $E_{1}$ from 20 to 25 to $30 \mathrm{eV}$, the $s$ curves shift to the right from black to red to blue. As the $s$ curve shifts to the right, $E_{\text {land }}$ will stay on the upper branch until this ceases to exist at $E_{0}=25 \mathrm{eV}$. At that point, $E_{\text {land }}$ jumps from the upper (black and red dots) to the lower branch (blue dot). Hence, charging changes sign abruptly upon a continuous shift of $E_{1}$ during electron exposure as shown by the solid black line in Fig. 4(c). Going from high to low $E_{1}$ [dashed black line, Fig. 4(c)], we meet a second unstable point at lower $E_{1}$, forming a hysteresis loop. In Fig. 4(d), the excursions of the $s$ curve are reduced as $g_{0}$ increases: the hysteresis loop shrinks as $g_{0}$ increases from 0.0025 to 0.005 [Fig. 4(c), blue lines]. Again, this is emblematic of a cusp catastrophe [11]. Of course, the experiment can only move from low to high $E_{1}$, as exposure-induced changes are irreversible. Figure 4(c) suggests that increasing $E_{1}$ causes the jumps in Fig. 2, while $g_{0}$ controls the height of the jumps.

Changes in SEE during electron exposure are well documented: bond breaking leads to creation of traps which capture secondary electrons and reduce SEE (upward shift of $E_{1}$ ) [22]. Similarly, trap creation leads to doping of the PMMA, and increases conductance (and thus $g_{0}$ ) during exposure. Increasing $E_{1}$ (i.e., decreasing SEE during exposure) causes the jump in Fig. 4(c), while increasing $g_{0}$ reduces the size of the jump.

Returning to Figs. 2(b)-2(e), the red lines are fits using Eq. (5b), linearly increasing $g_{0}$ and $E_{1}$ with time. In Fig. 2(b) we only change $g_{0}$, as the experiment (near threshold) is not very sensitive to $E_{1}$. Going from (b) to (e), both $g_{0}$ and $E_{1}$ increase with exposure. In Figs. 2(b) and 2(c) we start with $g_{0}=0.0025, E_{1}=18 \mathrm{eV}$, and in Fig. 2(e) we finish the experiment with $g_{0}=0.025$, $E_{1}=36 \mathrm{eV}$, i.e., a 10 -fold increase in $g_{0}$, and a twofold increase in $E_{1}$.

In Figs. 2(d)-2(e) we observe a drop in $E_{\text {land }}$ during the first few seconds that is not included in the fits. We now introduce a third phenomenon, ignored thus far: dielectric breakdown. In Fig. 2(d), the initial $E_{\text {land }}$ exceeds $40 \mathrm{eV}$, i.e., $V_{\text {eq }}>15 \mathrm{~V}$, corresponding to a field strength $>7.5 \mathrm{MV} / \mathrm{cm}$. In Fig. 2(e), with an initial $V_{\text {eq }}>25 \mathrm{~V}$, the field strength exceeds $12.5 \mathrm{MV} / \mathrm{cm}$. (These initial landing energies are again consistent with starting values of $g_{0} \approx 0.0025$ and $E_{1} \approx 18 \mathrm{eV}$.) In Fig. 2(c) the initial field strength is $\approx 5 \mathrm{MV} / \mathrm{cm}$, in Fig. 2(b) $\sim 7 \mathrm{MV} / \mathrm{cm}$. Thus, the dielectric breakdown strength exceeds $7 \mathrm{MV} / \mathrm{cm}$, a factor 2 larger than previous observations [25]. At higher fields 
(and assisted by incident electrons with $E_{\text {land }}>40 \mathrm{eV}$ ), the PMMA films undergo dielectric breakdown, until they become sufficiently conductive to reduce the charging potential, thereby stopping breakdown. In Figs. 2(d)-2(e) the film stabilizes after a few seconds at a charging potential of $\sim 5 \mathrm{eV}$, leading to $E_{\text {land }}=30$ and $35 \mathrm{eV}$, respectively. Now the normal charging and exposure processes take over, and the data are well described by Eq. (5b), as shown by the fits in Figs. 2(d)-2(e).

The rates of change in $g_{0}$ and $E_{1}$ don't depend strongly on electron energy. In Fig. 2(b) where $E_{\text {land }} \approx 0, g_{0}$ increases only a factor of 2 slower than for $E_{\text {land }} \approx$ $30 \mathrm{eV}$ [Fig. 2(c)]. In Figs. 2(d)-2(e) the rates are somewhat smaller, after the initial breakdown-induced changes.

With some of the basic processes already previewed in Fig. 1(c), our analysis reveals a wealth of information: (i) During electron exposure the PMMA surface charges due to limited conductance. (ii) Charging can be either positive or negative, with a change in sign when $E_{0} \approx E_{1}$ [Fig. 4(c)]. The initial value of $E_{1} \approx 18 \mathrm{eV}$ [Figs. 2(b), 2(c)] is more than 3 times lower than the literature suggests [17], and increases during electron exposure. (iii) Changes in $g_{0}$ and $E_{1}$ during exposure result in charging instabilities with a sudden change in the sign of $V$ when $E_{1}$ becomes greater than $E_{0}$. A cusp catastrophe in Eqs. (5) is responsible for these instabilities. (iv) Electrons with $E_{\text {land }} \approx 0 \mathrm{eV}$ expose PMMA [Fig. 2(b)]; $g_{0}$ increases at roughly the same rate for $E_{\text {land }} \approx 0 \mathrm{eV}$, as for $15-35 \mathrm{eV}$. Dissociative electron attachment likely plays an important role in determining line edge roughness (LER), proximity effects, dose efficiency, and other effects in EUV and electron lithography. (v) At field strengths $>7.5 \mathrm{MV} / \mathrm{cm}$ (20 nm PMMA) dielectric breakdown gives rise to a rapid increase in $g_{0}$ and $E_{1}$ during the first few seconds of exposure.

While these results are for PMMA, experiments on exploratory EUV resists containing $\mathrm{Sn}$ (oxo) cages $[26,27]$ display the same effects: sensitivity for near-zero $\mathrm{eV}$ electrons and strong charging instabilities. As the same basic mechanisms apply, the experimental and theoretical methodology developed here will make it possible to study such EUV resists more fully, and to contribute directly to their characterization and optimization. Surface charging, resist conductance, secondary electron emission, charging instabilities, and dielectric breakdown are not routinely considered in simulations of resist exposure, nor is the role of low electron energy processes such as dissociative electron attachment $[28,29]$. We suggest that these effects can no longer be ignored. During spatially patterned illumination charging will also vary spatially and electric fields will develop both normal and parallel to the surface. This will have additional ramifications for pattern definition and LER in lithography applications.

With excellent low energy beam control and built-in spectroscopic capabilities, LEEM offers a powerful new approach for studying and understanding the interactions of low energy electrons with organic materials. The ability to observe the electron energy loss spectrum during irradiation gives real-time access not only to charging phenomena, but also to changes in secondary electron emission, inelastic energy losses [30], and even electronic band structure [31,32]. Future studies of resist materials, as well as other organic and inorganic films, including biological materials such as DNA, organic semiconductors and metals, will greatly expand and enrich our understanding of the interaction of low energy electrons with soft matter of all kinds.

This work has received financial support from the Netherlands Organization for Scientific Research (NWO), domain Technical and Applied Sciences (TTW), ASML (Veldhoven, Netherlands), and SPECS Surface Nano Analysis GmbH (Berlin, Germany). The authors thank Joost Frenken and Fred Brouwer (Advanced Research Center for Nanolithography, Amsterdam, Netherlands), Wim van der Zande (ASML, Veldhoven, The Netherlands) and Jim Hannon (IBM T. J. Watson Research Center, Yorktown Heights, NY) for insightful discussions and suggestions. We thank Marcel Hesselberth and Daan Boltje for their technical support.

[1] EUV Lithography edited by V. Bakshi (SPIE and John Wiley \& Sons, New York, 2009), ISBN 9780819469649/ 9780470471555.

[2] Ernst Bauer, Surface Microscopy with Low Energy Electrons (Springer, New York, 2014), ISBN 978-1-49390934-6.

[3] R. M. Tromp, M. Mankos, M. C. Reuter, A. W. Ellis, and M. Copel, Surf. Rev. Lett. 05, 1189 (1998).

[4] A. Thete, D. Geelen, S. Wuister, S. J. van der Molen, and R. M. Tromp, Proc. SPIE 9422, Extreme EUV Lithography 94229A-94220A-5, (2015); S. M. Schramm, J. Kautz, A. Berghaus, O. Schaff, R. M. Tromp, and S. J. van der Molen, IBM J. Res. Dev. 55, 1:1 (2011).

[5] O. Wollersheim, H. Zumaqué, J. Hormes, D. Kadereit, J. langen, L. Häußling, P. Hoessel, and G. Hoffmann, Nucl. Instrum. Methods Phys. Res., Sect. B 97, 273 (1995).

[6] H. Hiraoko, IBM J. Res. Dev. 21, 121 (1977).

[7] E. M. Lehockey and I. Reid, J. Vac. Sci. Technol. A 6, 2221 (1988).

[8] B. W. Yates and D. M. Shinozaki, J. Polym. Sci., Part B: Polym. Phys. 31, 1779 (1993).

[9] The Radiation Chemistry of Macromolecules Volumes I and II, edited by M. Dole (Academic Press, New York, 1972).

[10] S. Rangan, R. A. Bartynski, A. Narasimhan, and R. L. Brainard, J. Appl. Phys. 122, 025305 (2017).

[11] R. Gilmore, Catastrophe Theory for Scientists and Engineers (John Wiley \& Sons, New York, 1981), ISBN 0-471-05064-4.

[12] M. Braun, F. Gruber, M.-W. Ruf, S. V. K. Kumar, E. Illenberger, and H. Hotop, Chem. Phys. 329, 148 (2006). 
[13] R. M. Tromp, Y. Fujikawa, J. B. Hannon, A. W. Ellis, A. Berghaus, and O. Schaff, J. Phys. Condens. Matter 21, 314007 (2009).

[14] N. F. Mott and R. W. Gurney, Electronic Processes in Ionic Crystal, 1st ed. (Oxford University Press, Oxford, 1940); See also: M. Pope and C. E. Swenberg, Electronic Processes in Organic Crystals and Polymer, 2nd ed. (Oxford University Press, Oxford, 1999).

[15] D. C. Joy, M. S. Prasad, and H. M. Meyer III, J. Microsc. 215, 77 (2004).

[16] J. Cazaux, Nucl. Instrum. Methods Phys. Res., Sect. B 244, 307 (2006).

[17] E. A. Burke, IEEE Trans. Nucl. Sci. NS-27, 1760 (1980).

[18] J. J. Scholtz, D. Dijkkamp, and R. W. A. Schmitz, Philips J. Res. 50, 375 (1996).

[19] Y. Lin and D. C. Joy, Surf. Interface Anal. 37, 895 (2005).

[20] K. Said, G. Damamme, A. SI Ahmed, G. Moya, and A. Kallel, Appl. Surf. Sci. 297, 45(2014).

[21] J. P. Ganachaud and A. Mokrani, Surf. Sci. 334, 329 (1995).

[22] M. Dapor, M. Ciappa, and W. Fichtner, J. Micro/ Nanolithogr. MEMS MOEMS 9, 023001 (2010).

[23] A. Dunaevsky, Y. Raitses, and N. J. Fisch, Phys. Plasmas 10, 2574 (2003), and references therein.

[24] See Supplemental Material at http://link.aps.org/ supplemental/10.1103/PhysRevLett.119.266803 for more detailed information on (i) Secondary Electron Emission, (ii) The insensitivity of the result to the value of $\alpha$ in Eq. (4), (iii) Various solutions to Eq. (5b), (iv) The cusp catastrophe contained in Eq. (5b), and (v) Experimental details, including native oxide, and current measurements.

[25] C. Neusel and G. A. Schneider, J. Mech. Phys. Solids 63, 201 (2014).

[26] B. Cardineau, R. Del Re, H. Al-Mashat, M. Marnell, M. Vockenhuber, Y. Ekinci, C. Sarma, M. Neisser, D. A. Freedman, and R. L. Brainard, Proc. SPIE 9051, Advances in Patterning Materials and Processes XXXI, 90511B (2014).

[27] A. Thete, D. Geelen, Y. Zhang, J. Haitjema, F. Brouwer, S. J. van der Molen, and R. M. Tromp (to be published).

[28] L. Wisehart, A. Narasimhan, S. Grzeskowiak, M. Neisser, L. E. Ecola, G. Denbeaux, and R. L. Brainard, Proc. SPIE 9776, Extreme Ultraviolet (EUV) Lithography VII, 97762 O (2016).

[29] A. Narasimhan, S. Grzeskowiak, B. Srivats, H. Herbol, L. Wisehart, C. Kelly, W. Early, L. E. Ocola, M. Neisser, G. Denbeaux, and R. L. Brainard, Proc. SPIE 9422, Extreme Ultraviolet (EUV) Lithography VI, 942208 (2015).

[30] Y. Fujikawa, T. Sakurai, and R. M. Tromp, Phys. Rev. Lett. 100, 126803 (2008).

[31] H. Hibino, H. Kageshima, F.-Z. Guo, F. Maeda, M. Kotsugi, and Y. Watanabe, Appl. Surf. Sci. 254, 7596 (2008).

[32] J. Jobst, J. Kautz, D. Geelen, R. M. Tromp, and S. J. van der Molen, Nat. Commun. 6, 8926 (2015). 